\title{
Congenital Pulmonary Agenesis of Left Upper Lobe
}

\author{
PROBIR KUMAR SARKAR ${ }^{1}$, NABILAAKAND ${ }^{2}$, MD. SHAKIBUR RAHMAN ${ }^{3}$, MD. KAMRUZZAMAN ${ }^{4}$
}

\begin{abstract}
Underdevelopment of lung is a group of congenital anomalies classified into agenesis, aplasia, and hypoplasia of lung, on the basis of presence or absence of lung parenchyma, bronchial tree, and pulmonary artery. The pathogenesis of such anomalies is not accurately known with several proposed theories. It is often associated with anomalies of the cardiovascular \& genitourinary systems. An isolated left upper lobe pulmonary agenesis is rare and more commonly involves the right upper and middle lobes. The prevalence of this condition has been noted to be $0.0034-0.0097 \%$. It is associated with the absence of lung parenchyma, bronchial tree, pulmonary vasculature, and may remain undiagnosed until adulthood. Treatment depends on patient's symptoms, with most patients being treated conservatively. Herein, we report an eight month old infant who presented with fever, cough, and respiratory distress and treated as a case of left sided pneumonic collapse consolidation without radiological improvement and finally diagnosed as a case of left upper lobe pulmonary agenesis with low serum IgA level.
\end{abstract}

Keywords: Congenital anomaly, lung agenesis, aplasia, and hypoplasia of lung.

\section{Introduction}

Pulmonary agenesis is a very rare congenital abnormality that represents the unsuccessful growth of the primitive lung bud. ${ }^{1}$ Agenesis means complete absence of bronchus, lung parenchyma and associated vessels. ${ }^{2}$ Bilateral pulmonary agenesis is incompatible with life, manifesting as severe respiratory distress and failure. Pulmonary agenesis is thought to be an autosomal recessive trait, with an estimated incidence of 1 in 10,000-15,000 births. ${ }^{3}$

The pathogenesis of such anomalies is not accurately known with several proposed theories. Almost half of pulmonary agenesis cases accompany congenital defects, which may involve multiple organ systems. ${ }^{4}$ It is often associated with anomalies of the cardiovascular, genitourinary systems. An isolated left upper lobe pulmonary agenesis is rare and more commonly involves the right upper and middle lobes. ${ }^{5}$ We describe here an infant who was shown to have isolated pulmonary agenesis of the left upper lobe with low serum IgA level.

1. Associate Professor \& Head, Pediatric Pulmonology, Bangladesh Institute of Child Health \& Dhaka Shishu (Children) Hospital.

2. Resident Medical Officer (RMO), Bangladesh Institute of Child Health \& Dhaka Shishu (Children) Hospital, Dhaka

3. FCPS Pediatric Pulmonology Trainee, Bangladesh Institute of Child Health \& Dhaka Shishu (Children) Hospital, Dhaka

4. Assistant Professor, Pediatric Pulmonology, Bangladesh Institute of Child Health \& Dhaka Shishu (Children) Hospital, Dhaka

Correspondence: Dr. Probir Kumar Sarkar, Associate Professor \& Head, Department of Pediatric Pulmonology, Bangladesh Institute of Child Health \& Dhaka Shishu (Children) Hospital. Email: drprobirdsh@ gmail.com, Mobile: 01711225099.

Received: 03-01-2018 Accepted: 24-06-2019

\section{Case Report}

Farhan, an 8 months old immunized boy only issue of his non-consanguineous parents presented with the complaints of cough and cold for 1 month, respiratory distress for 5 days and high grade intermittent fever for 2 days. He had 2 similar episodes of illness at 20 days of age and again at 3 months which had resolved very shortly after treatment with parenteral antibiotics. There was no history of prolong fever, evening rise of temperature, loss of appetite or weight loss, contact with any TB patient and $\mathrm{H} / \mathrm{O}$ choking. On examination, the child was dyspneic, tachypneic, R/R-60/min, chest indrawing was present, mildly pale, afebrile, pulse rate-140/min, BCG mark was present. Chest movement was restricted on the left side with mediastinum shifted to the left, air entry was diminished on the left side up to the $2^{\text {nd }}$ intercostal space but normal on the rest of the lung fields and breath sound was vesicular with crepitations on both lung fields. There was no lymphadenopathy and organomegaly.

CBC values revealed $\mathrm{Hb} \% 9 \mathrm{~g} / \mathrm{dl}$, Total count of leukocyte- $17,500 / \mathrm{mm}^{3}$, differential count of leukocyte- neutrophil $75 \%$, lymphocyte $20 \%$, monocyte $4 \%$, eosinophil $1 \%$. The serial chest radiography showed persistent opacity of the left hemithorax along with mediastinal shifting evidenced by shifting of the trachea and the heart and there was compensatory hyperinflation of the right lung field, may be due to left sided collapse-consolidation. 


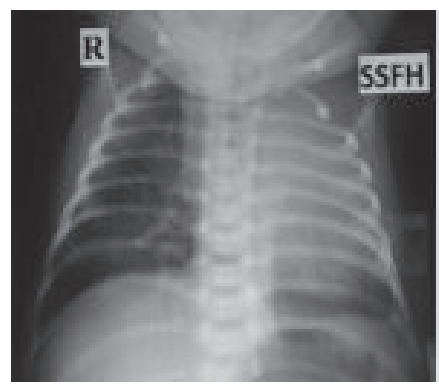

CXR-1(a): 25days : 25/05/2017

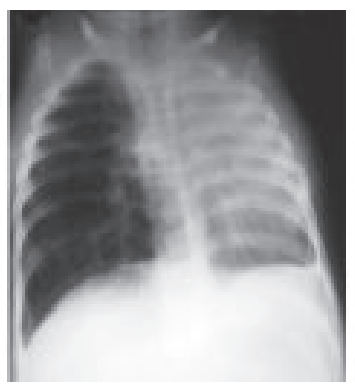

CXR-1(b): 3 months: 12/07/2017

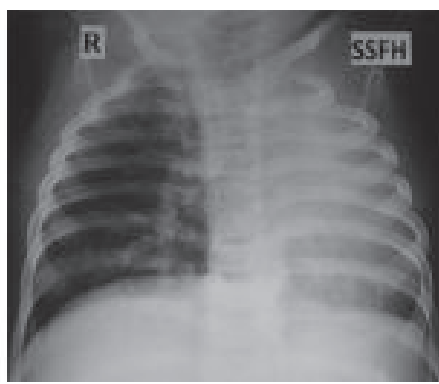

CXR-1(c): 7 months : 20/11/2017

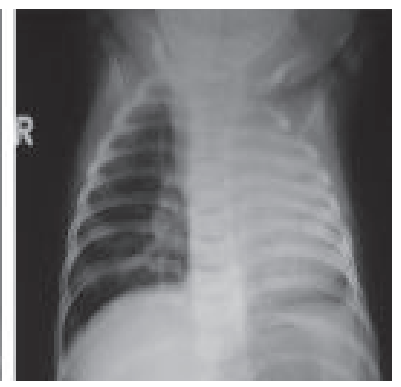

CXR-1(d): 8months : $0 / 12 / 2017$
Pulmonary tuberculosis was excluded with negative MT $(03 \mathrm{~mm}$ in $72 \mathrm{hrs}$ ) and gastric lavage for AFB staining and Gene Xpert showing no Mycobacterium tuberculosis detected. Then, contrast enhanced chest CT (CECT) scan was performed. CECT chest revealed the absence of left upper and lingular bronchus with complete agenesis of the left upper lobe. Compensatory hyperinflation with herniation of the right lower lobe to the left side was also noted. After contrast, no abnormal enhancement of the lesion was seen.

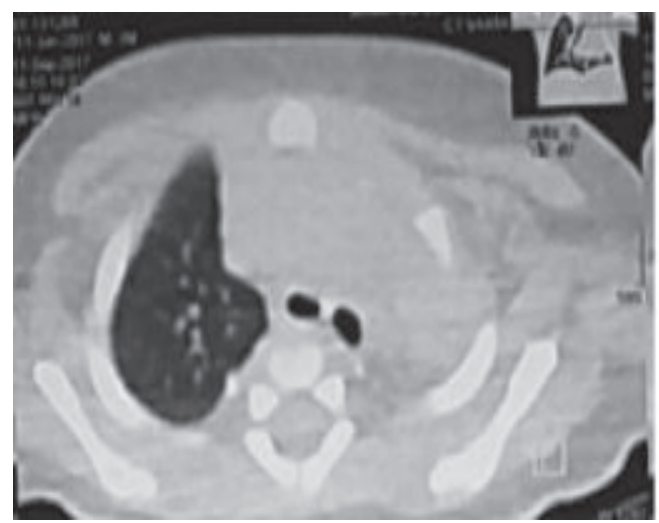

CECT Chest: pulmonary window

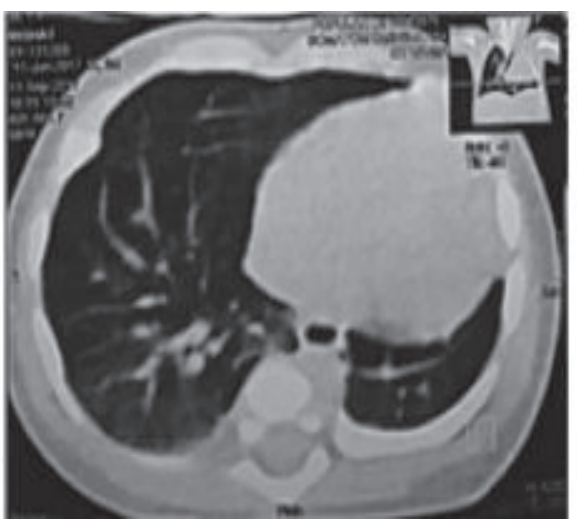

CECT Chest: pulmonary window

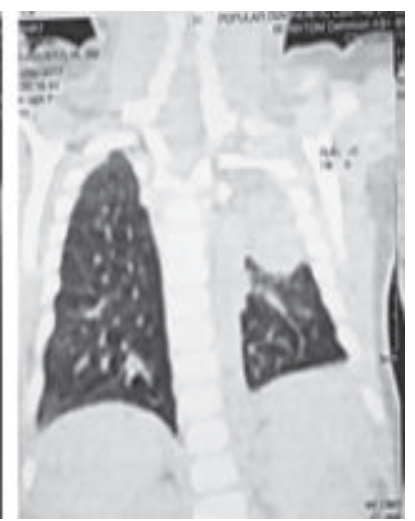

CECT Chest: coronal view

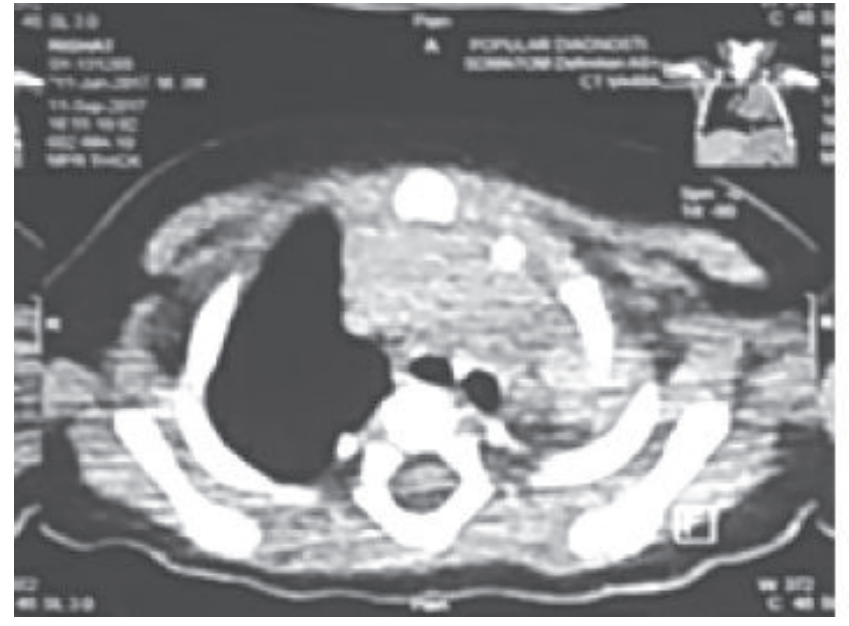

CECT Chest: Mediastinal window

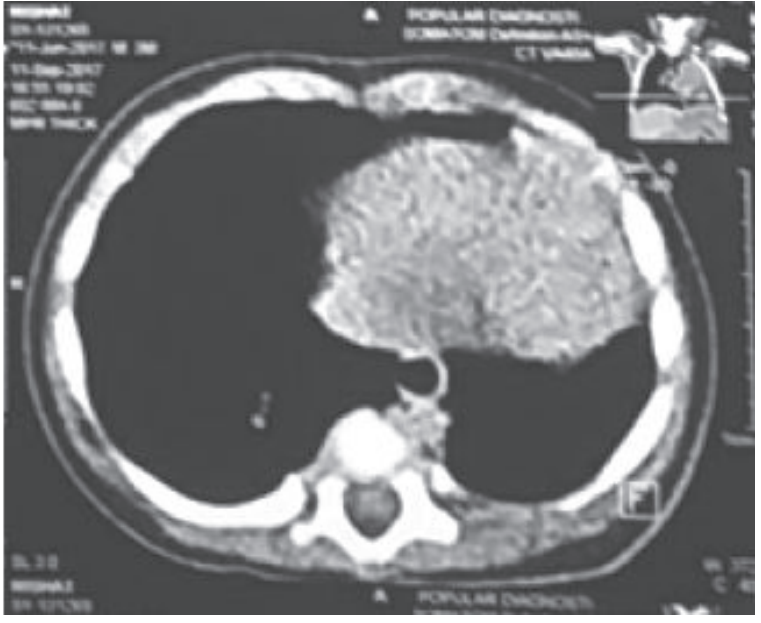

CECT Chest: mediastinal window 
No other associated congenital anomaly was found on color droppler echocardiography. Immunoglobulins assay was done which showed low level of IgA (IgA: $0.64 \mathrm{~g} / \mathrm{L}$; normal $0.7-4.0 \mathrm{~g} / \mathrm{L})$. Finally, the patient was diagnosed as a case of congenital pulmonary agenesis of left upper lobe with low serum IgA level.

He was initially treated with parenteral antibiotics for infection along with other supportive care for 7 days and opinion from cardio-thoracic surgeon was taken and he had advised to manage the child conservatively with routine follow up to 8 years of age. Counselling was done to his parents about the nature of his disease and thereafter was discharged with advice. Now the child is on routine follow up and thriving well.

\section{Discussion}

Pulmonary agenesis is one the rarest congenital disorders of the lung. The other forms of congenital disorders of the lung includes aplasia, hypoplasia, congenital cystic malformation, pulmonary sequestration, bronchogenic cysts, lung hernia, congenital pulmonary lymphangiectasia, congenital lobar emphysema and pulmonary cyst, arteriovenous malformation. Among them some authors have classified 'underdevelopment of lung' into three categories:

1. Agenesis shows complete absence of bronchus, lung parenchyma and associated vessels

2. Aplasia has a rudimentary bronchus with absent lung parenchyma

3. Hypoplasia has hypoplastic bronchus and lung parenchyma. ${ }^{6}$

It differs from hypoplasia in that agenesis entails the complete absence of a lung. Agenesis differs from aplasia by the absence of a bronchial stump or carina that is seen in aplasia. ${ }^{3}$ Pulmonary agenesis has been classified morphologically based on the extent to which broncho-pulmonary tissue is absent. Spencer modified earlier classification and divided pulmonary agenesis into three categories: (1) bilateral complete agenesis; (2) unilateral agenesis; and (3) lobar agenesis or lesser forms of congenital anomaly. ${ }^{7}$

The etiopathogenesis is unclear; however, genetic, teratogenic agents (allopurinol), and Vitamin A deficiency during pregnancy have been hypothesized as its causes. ${ }^{8,9}$
The abnormality goes undetected or is incidentally detected in the majority of cases. Symptomatic patients present in early childhood with symptoms of recurrent respiratory tract infection while others remain asymptomatic. Underdevelopment of lung is usually associated with other congenital abnormalities of cardiovascular, musculoskeletal, and genitourinary system, more commonly toward the ipsilateral side. Mardini-Nyhan association is seen in consanguineous marriages, comprising congenital heart disease, thumb abnormality, and complete/ partial lung agenesis. ${ }^{10}$ Our case had no other commonly found associated congenital abnormality but IgA deficiency.

Genetic defects of the pulmonary system and embryonic disorders of the bronchi contribute to anomaly, but the pathogenesis remains controversial. Less than $5 \%$ of the general population develop bronchial anomalies, and the malformation of the right upper bronchus is the most common abnormality. Pulmonary artery hypoplasia is commonly accompanied with such an anomaly of the lung lobe. ${ }^{11}$ An investigation of the associated vascular abnormalities is therefore necessary.

Isolated lobar agenesis is usually asymptomatic and may remain undiagnosed throughout childhood until a referral for an abnormal chest radiograph. ${ }^{12}$ In left upper lobe agenesis of the lung, the presentations of a chest radiograph include a decreased left lung volume, shifting of the mediastinum on the left side, and an elevation of the left hemidiaphragm. The differential diagnosis of our patient's chest radiograph includes left upper lobe atelectasis, left hemi diaphragmatic eventration, pulmonary hypoplasia/ agenesis and left sub-pulmonic effusion. A chest CT is considered to be the most conclusive examination to diagnose congenital abnormalities of the lung and the associated vascular anomalies when a chest radiograph is not diagnostic. ${ }^{13}$ Three-dimensional reconstruction can be particularly helpful in delineating abnormalities of the bronchi and associated arterial and venous structures. Angiography is reserved for patients requiring embolization or revascularization surgery. Treatment is necessary for recurrent chest infections. The prognosis is good if there is no other malformation. ${ }^{2}$ 


\section{Conclusion}

A patient who has been consecutively demonstrating an abnormal chest radiograph with or without respiratory symptoms, we should always consider a congenital anomaly of the lung. A chest CT may be useful in determining the extent of the lesion and to define associated abnormalities.

\section{References}

1. Mardini MK. Nyhan WL. Agenesis of the lung: report of four patients with unusual anomalies. Chest 1985;87:522-527.

2. Gupta Keshav, Taneja Dhruv, Aggarwal Monika and Gupta Rajiv. Left upper lobe agenesis of lung: A rare case report. Lung India 2017 34(3): 302-304.

3. Joshua A. Blatter and Jonathan D. Finder editor. Nelson Textbook of Paediatrics: Congenital Disorders of the Lung. 20 ${ }^{\text {th }}$ Edition. Vol 2. Philadelphia: Elsevier; 2016. p. 2057.

4. Chiu-Ping Kuo, Yen-Ta Lu \& Rong-Luh Lin Agenesis of right upper lobe. Respiratory Case Reports 2015;3(2): 51-53.

5. Clements BS. Congenital malformations of the lungs and airways. In: Landau LI, Taussing LM, editors. Pediatric Respiratory Medicine. St. Louis, MO, USA: Mosby; 1999. pp. 1106-36.

6. Berrocal T, Madrid C, Novo C, Gutiërrez J, Arjonilla A, Gymez-Leyn N. Congenital anomalies of the tracheobronchial tree, lung, and mediastinum: Embryology, radiology and pathology. Radiographics 2004;24: e17.

7. Schwartz M, Ramchandran P. Congenital malformation of the lung and mediastinum- a quarter century of experience from a single institution. J.Pediatr. Surg. 32: 44-47.

8. Kozenko M, Grynspan D, Oluyomi-Obi T, Sitar $D$, Elliott AM, Chodirker BN. Potential teratogenic effects of allopurinol: A case report. Am J Med Genet A. 2011;155A:224752.

9. Kisku KH, Panigrahi MK, Sudhakar R, Nagarajan A, Ravikumar R, Daniel JR. Agenesis of lung - A report of two cases. Lung India 2008; 25:28-30.

10. Hastings $R$, Harding D, Donaldson A, Liebling $R$, Hayes A, Kraus A, et al. Mardini-Nyhan association (lung agenesis, congenital heart, and thumb anomalies): Three new cases and possible recurrence in a sib-is there a distinct recessive syndrome? Am J Med Genet A. 2009;149A:2838-42.

11. Stovin PGI. Early lung development. Thorax. 1985; 40: 401-405.

12. Zack MS. Eber E. Adult outcome of congenital lower respiratory tract malformations. Thorax. 2001; 56:65-72.

13. Kuo CP, Lu YT, Lin RL. Agenesis of right upper lobe of lung. Respirol Case Rep. 2015; 3:51-3. 